A. Е. Вязилов, Остаточная эмпирическая функция распределения в $\operatorname{GARCH}(1,1)$ и ее применение при проверке гипотез, УМН, 1999, том 54, выпуск 4, 163-164

DOI: https://doi.org/10.4213/rm195

Использование Общероссийского математического портала Math-Net.Ru подразумевает, что вы прочитали и согласны с пользовательским соглашением

http://www.mathnet.ru/rus/agreement

Параметры загрузки:

IP: 54.80 .73 .141

26 апреля 2023 г., 17:04:48 


\title{
ОСТАТОЧНАЯ ЭМПИРИЧЕСКАЯ ФУНКЦИЯ РАСПРЕДЕЛЕНИЯ В GARCH(1,1) И ЕЕ ПРИМЕНЕНИЕ ПРИ ПРОВЕРКЕ ГИПОТЕЗ
}

\author{
А. Е. Вязилов
}

1. Модель GARCH $(1,1)$ задается соотношениями

$$
y_{k}=\sigma_{k} \varepsilon_{k}, \quad \sigma_{k}^{2}=\alpha+\beta y_{k-1}^{2}+\gamma \sigma_{k-1}^{2} ; \quad k=0, \pm 1, \ldots,
$$

где $\left\{\varepsilon_{k}\right\}$ - независимые одинаково распределенные случайные величины с неизвестной функцией распределения $G(x), \varepsilon_{1}^{2}=1 ; \mathbf{a}=(\alpha, \beta, \gamma)$ - вектор неизвестных параметров, $\alpha, \beta, \gamma>0$. Если $\beta+\gamma<1$, то система (1) имеет единственное стационарное решение

$$
y_{k}=\alpha^{1 / 2} \varepsilon_{k}\left[1+\sum_{m=1}^{\infty} \prod_{i=1}^{m}\left(\beta \varepsilon_{k-i}^{2}+\gamma\right)\right]^{1 / 2},
$$

при этом $\mathrm{E} y_{k}^{2}=\alpha[1-\beta-\gamma]^{-1}<\infty$.

Модель GARCH впервые была рассмотрена в [1] при условии, что $G(x)$ - нормальная функция распределения. В работе излагается способ проверки общей гипотезы $\Gamma_{0}: G(x)=G_{0}(x)$, в том числе гипотезы нормальности. Этот тест основан на асимптотической равномерной линейности остаточной эмпирической функции распределения (см. теорему ниже). Для моделей с аддитивными шумами общие результаты такого типа получены для линейных [2] и нелинейных [3] моделей. Подход этой работы является обобщением на модель GARCH методов, предложенных для схем МА и $\mathrm{ARCH} \mathrm{в} \mathrm{[4],} \mathrm{[5].}$

2. Пусть $y_{0}, \ldots, y_{n}-$ наблюдения из стационарного решения схемы (1). Положим

$$
\sigma_{k}^{2}(\boldsymbol{\theta}):=\theta_{1}+\theta_{2} y_{k-1}^{2}+\theta_{3} \sigma_{k-1}^{2}(\boldsymbol{\theta}), \quad \varepsilon_{k}(\boldsymbol{\theta}):= \begin{cases}\frac{y_{k}}{\sigma_{k}(\boldsymbol{\theta})}, & \text { если } \sigma_{k}(\boldsymbol{\theta})>0, \\ 1, & \text { если } \sigma_{k}(\boldsymbol{\theta}) \leqslant 0,\end{cases}
$$

где $k=1, \ldots, n, \boldsymbol{\theta}=\left(\theta_{1}, \theta_{2}, \theta_{3}\right), \sigma_{0}(\boldsymbol{\theta}) \equiv 0$. Введем для $\boldsymbol{x} \in \mathbb{R}, \boldsymbol{\theta} \in \mathbb{R}^{3}$ остаточную әмпирическую функцию распределения

$$
G_{n}(x, \boldsymbol{\theta}):=\frac{1}{n} \sum_{k=1}^{n} I\left\{\varepsilon_{k}(\boldsymbol{\theta}) \leqslant x\right\},
$$

являющуюся аналогом истинной эмпирической функции распределения $G_{n}(x)$.

Выпишем нужные нам условия на $G(x)$ :

(i) $G(x)$ - строго монотонна на множестве $S=\{x \in \mathbb{R}: 0<G(x)<1\}$,

(ii) сушествует плотность $g(x)=G^{\prime}(x)$ и ее производная $g^{\prime}(x)$, такая что $\sup _{x \in \mathbb{R}} x^{2}\left|g^{\prime}(x)\right|<\infty$.

Обозначим $\mathrm{e}=\left(\mathrm{E} \frac{X}{\alpha X+\beta Y}, \mathrm{E} \frac{Y}{\alpha X+\beta Y}, \mathrm{E} \frac{Z}{\alpha X+\beta Y}\right)$, где $X=\frac{1}{1-\gamma}, Y=\sum_{m=0}^{\infty} \gamma^{m} y_{-m}^{2}$, $Z=\frac{\alpha}{(1-\gamma)^{2}}+\sum_{m=1}^{\infty} m \gamma^{m-1} y_{-m}^{2}$ и эти ряды сходятся в $\mathrm{L}_{1}$. Пусть $\langle\cdot, \cdot\rangle$ - евклидово скалярное произведение и $\mathbf{t} \in \mathbb{R}^{3}$.

Теорема. Если выполнены условия (i) и (ii), то для любого $T \geqslant 0$ при $n \rightarrow \infty$

$$
\sup _{x \in \mathbb{R},|\mathbf{t}| \leqslant T}\left|\sqrt{n}\left[G_{n}\left(x, \mathbf{a}+\frac{\mathbf{t}}{\sqrt{n}}\right)-G_{n}(x)\right]-\frac{1}{2} x g(x)\langle\mathbf{t}, \mathbf{e}\rangle\right|=o_{p}(1) .
$$

Заметим, что для ARCH (2) совпадает с утверждением теоремы 1.1 из [5]. Пусть $\widehat{\mathbf{a}}_{n}$ есть $\sqrt{n}$-состоятельная оценка вектора параметров а (см. [6], [7]). Обозначим $\widehat{G}_{n}(x):=G_{n}\left(x, \widehat{\mathbf{a}}_{n}\right)$. Из теоремы получаем 
СЛЕДСТвИЕ 1. Пусть выполнены условия (i), (ii) $u \sqrt{n}\left(\widehat{\mathbf{a}}_{n}-\mathbf{a}\right)=O_{p}(1)$. Тогда при $n \rightarrow \infty$

$$
\sup _{x \in \mathbb{R}}\left|\sqrt{n}\left[\widehat{G}_{n}(x)-G_{n}(x)\right]-\frac{1}{2} x g(x)\left\langle\sqrt{n}\left(\widehat{\mathbf{a}}_{n}-\mathbf{a}\right), \mathbf{e}\right\rangle\right|=o_{p}(1) .
$$

3. Полученнный результат можно применить для проверки гипотезы $\Gamma_{0}: G(x)=G_{0}(x)$, где $G_{0}(x)$ - известная функция распределения. Аналогичный тест использовался в [5] для проверки гипотезы $\Gamma_{0}$ в схеме ARCH. Его построение основано на идеях [8].

Пусть $\mathrm{C}:=\left\{H(t), t \in[0,1]: H(0)=0\right.$ и существует $\left.H^{\prime}(t) \in \mathrm{L}_{2}[0,1]\right\}$. Введем скалярное произведение $\left(H_{1}, H_{2}\right):=\int_{0}^{1} h_{1}(t) h_{2}(t) d t$, где $H_{i} \in \mathrm{C}, h_{i}(t)=H_{i}^{\prime}(t)$. Положим $\widehat{U}_{n}(t):=$ $\sqrt{n}\left[\widehat{G}_{n}\left(G_{0}^{-1}(t)\right)-t\right]$ и $\left(H_{i}, \widehat{U}_{n}\right)=\int_{0}^{1} h(t) d \widehat{U}_{n}(t):=n^{-1 / 2} \sum_{k=1}^{n} h\left(G_{0}\left(\varepsilon_{k}\left(\widehat{\mathbf{a}}_{n}\right)\right)\right)-\sqrt{n} \int_{0}^{1} h(t) d t$. Обозначим $a_{0}(t):=G_{0}^{-1}(t) g_{0}\left(G_{0}^{-1}(t)\right)$, и пусть $a_{0} \in$ С. Выберем функции $\left\{H_{i}, i=1,2, \ldots\right\} \in$ С, удовлетворяющие условию

(iii) $\left(H_{i}, H_{j}\right)=\delta_{i j},\left(H_{i}, t\right)=0,\left(H_{i}, a_{0}\right)=0, H_{i}^{\prime \prime} \in \mathrm{L}_{1}[0,1]$.

В качестве тестовой статистики для проверки $\Gamma_{0}$ возьмем $\Xi_{m}^{2}:=\sum_{i=1}^{m}\left(H_{i}, \widehat{U}_{n}\right)^{2}$. Асимптотическое разложение для $\widehat{U}_{n}(t)$, вытекающее из следствия 1 и условия (iii), влечет

СлЕДСтвиЕ 2. Если выполнены условия (i)-(iii), $a_{0} \in \mathrm{C}, \sqrt{n}\left(\widehat{\mathbf{a}}_{n}-\mathbf{a}\right)=O_{p}(1) u$ иипотеза $\Gamma_{0}$, то $\Xi_{m}^{2}=\sum_{i=1}^{m}\left[n^{-1 / 2} \sum_{k=1}^{n} h_{i}\left(G_{0}\left(\varepsilon_{k}\right)\right)\right]^{2}+o_{p}(1) \stackrel{d}{\rightarrow} \chi^{2}(m)$, где $\chi^{2}(m)-$ распределение хи-квадрат с т степенями свободь.

Например, при проверке гипотезы $\Gamma_{\Phi}: G(x)=\Phi(x)$, где $\Phi(x)$ - гауссовская функция распределения, можно взять $h_{i}(\Phi(x))=c_{i}^{-1} P_{i}(x / \sqrt{2}), i=1,3,4, \ldots$, где $P_{i}(x)$ - полиномы Эрмита, а $c_{i}^{2}=\frac{1}{\sqrt{\pi}} \int_{-\infty}^{\infty} P_{i}^{2}(x) e^{-x^{2}} d x$.

4. Рассмотрим гипотезу симметрии $\Gamma_{s}: G(x)+G(-x)=1$. Тестовой статистикой для проверки $\Gamma_{s}$ возьмем $\widehat{\omega}_{n}^{2}:=n \int_{-\infty}^{\infty}\left[\widehat{G}_{n}(x)+\widehat{G}_{n}(-x)-1\right]^{2} d \widehat{G}_{n}(x)$. Исползуя нечетность функции $x g(x)$ при гипотезе $\Gamma_{s}$, из следствия 1 получаем, что

$$
\sup _{x \in \mathbb{R}}\left|\sqrt{n}\left[\widehat{G}_{n}(x)+\widehat{G}_{n}(-x)-1\right]-\sqrt{n}\left[G_{n}(x)+G_{n}(-x)-1\right]\right|=o_{p}(1) .
$$

Из (3) следует, что

$$
\widehat{\omega}_{n}^{2}=\omega_{n}^{2}+o_{p}(1)
$$

где статистика $\omega_{n}^{2}=n \int_{-\infty}^{\infty}\left[G_{n}(x)+G_{n}(-x)-1\right]^{2} d G_{n}(x)$ (см. [9]) имеет предельное распределение величины $\int_{0}^{1} w^{2}(t) d t$, где $w(t)$ - винеровский процесс. Оно затабулировано в [10] и в силу (4) совпадает с предельным распределением $\widehat{\omega}_{n}^{2}$ при гипотезе $\Gamma_{s}$.

Автор выражает признательность М. В. Болдину за постановку задачи и ее обсуждение.

\section{СПИСОК ЛИТЕРАТУРЫ}

[1] Bollerslev T. // J. Econometrics. 1986. V. 31. № 3. P. 307-327. [2] Koul H. L. Weighted Empirical and Linear Models. Hayward, CA: IMS, 1994. [3] Koul H. L. // Ann. Statist. 1996. V. 24. P. 380-404. [4] Болдин М. В. // Теория вероятн. и ее примен. 1989. Т. 34. С. 758-764. [5] Boldin M. V. // Mitt. Math. Sem. Giessen. 1998. V. 235. P. 49-66. [6] Shepard N. // Time Series Models in Econometrics, Finance and Other Fields / ed. D. R. Cox, D. V. Hinkley, O. E. Barndorff-Nielsen. London: Chapman \& Hall, 1996. P. 1-55. [7] Lee S. W., Hansen B. E. // Econometric Theory. 1994. V. 10. Р. 29-52. [8] Хмаладзе Э. В. // Теория вероятн. и ее примен. 1979. Т. 24. С. 280-297. [9] Мартынов Г. В. Критерии омега-квадрат. М.: Наука, 1978. [10] Орлов А. И. // Теория вероятн. и примен. 1972. Т. 17. С. 372-377. 